\title{
Anastomotic Leaks after Colorectal Surgery: a Prognostic Score
}

\author{
Caziuc Alexandra1 ${ }^{1}$ Mironiuc A² \\ ${ }^{1}$ First Surgical Clinic, "Iuliu Hațieganu" University of Medicine and Pharmacy, Cluj-Napoca, Romania \\ 2 Second Surgical Clinic, "Iuliu Hațieganu" University of Medicine and Pharmacy, Cluj Napoca, Romania
}

\begin{abstract}
Introduction: Anastomotic leaks are one of the most dreaded complications in colorectal surgery. At the ground of this problem remain the nefarious prognostic over the patient's life, the difficulties in early diagnosis and the optimal choice of therapeutical methods. The goal of our research was to evaluate the significance of possible risk factors in the postoperative evolution of patients following colorectal interventions and to design a prognostic score.

Material and methods: We performed a retrospective study on 298 cases of patients with inflammatory, neoplastic or traumatic lesions for which there was chosen a digestive anastomosis including the colon. The presumed risk factors in our analysis were: age, comorbidity evaluated by Charlson Score, etiology, toxics, use of nonsteroidal anti-inflammatory substances or corticoids, parameters such as leucocytes, hemoglobin, urea, total seric bilirubin, glycaemia, albumins, neoadjuvant therapy, type of suture, quantity of fluids received intraoperatory. All data were analyzed using R-Commander.

Results: All parameters for which we demonstrated a statistical significance $(p<0.05)$ for an unfavorable evolution were included in a prognostic score, designed in correlation with the statistical relation between the factors. We obtained values that certified a strong association (0.75) between a high prognostic score and an increased number of anastomotic leaks.

Conclusions: The practical utility of the score is mainly for the anticipation of postoperative complications. A high level score underlines that prior to surgery it is essential to balance the biochemical abnormalities and to choose properly the moment and type of surgical intervention.
\end{abstract}

Keywords: colorectal surgery, anastomotic leak, prognostic score

Received: 11 June 2013 / Accepted: 12 February 2014

\section{Introduction}

Postoperative digestive fistulas are an important surgical complication, both in terms of the variety of the clinical manifestations that may occur, and in terms of the many factors that contribute to their occurrence. They are a vast issue due to their influence on the quality of life, as well as to their forensic implications. Despite recent advances in anaesthesia and intensive care, and the development of new techniques for digestive fistulas suture, their frequency remains at a worrying rate (ranging between 1.5 and 16\%, depending on the segment of the digestive tract studied) resulting in a poor prognosis by increasing the length of the hospital stay and mortality (by 8 to 10 times) $[1,2,3]$.

The studies published in the literature mention two categories of factors involved in digestive suture failure: local factors, which relate to the anastomotic technique (suture type and material, local vascularisation) and the local characteristics of the underlying disease (neoplastic infiltration, local irradiation, infection, intraperitoneal chemotherapy), as well as general factors, which consist of systemic abnormalities affecting the sutures (age, nutritional status, anaemia, diabetes, uraemia, use of certain drugs). Despite the numerous data published so far, there is no consensus regarding the role that each factor plays in the digestive

Correspondence to: Alexandra Caziuc

E-mail: alex.8610@gmail.com anastomosis healing process [1]. Most authors argue that the importance of systemic factors is secondary, and that the main role is played by the local conditions at the level of the anastomosis. Classically, in achieving anastomosis, the surgeon must always consider the English acronym S.N.A.P. (sepsis, nutrition, anatomy, therapeutic procedures) [2].

The objective of our study was to develop a score to quantify the risk of complications in digestive anastomoses involving the colon.

\section{Material and method}

To achieve this goal, we conducted a retrospective study in the Surgery Clinic no. 2 in Cluj-Napoca between January 2000 - December 2012. The study included all patients with inflammatory, neoplastic or traumatic conditions requiring digestive anastomosis involving the colon (ileocolic, colo-colic, colo-rectal). The study excluded patients with a second surgery, whose purpose was to solve anastomotic complications, patients whose case report forms were incomplete, those who were transferred to other clinics and could not be monitored, and those who died during hospitalization due to causes independent of the digestive disease which required anastomosis. Based on these criteria a total of 298 cases were selected.

In this study, external fistulas were defined as leakage of intestinal contents on the outside, and internal fistulas as 
Table I. Prognostic factors of anastomotic fistulas studied

\begin{tabular}{lccc}
\hline Parameter studied & $\begin{array}{c}\text { Anastomotic } \\
\text { fistula } \\
\mathrm{n}=23\end{array}$ & $\begin{array}{c}\text { Uncompli- } \\
\text { cated cases } \\
\mathrm{n}=275\end{array}$ & $\mathrm{p}$ value \\
\hline Gender: male & 15 & 158 & 0.09 \\
Age $>60$ years & 13 & 149 & $0.04^{*}$ \\
Malignant aetiology & 15 & 190 & $0.03^{*}$ \\
Charlson score $>5$ & 17 & 198 & $0.04^{*}$ \\
Alcohol consumption & 7 & 197 & 0.14 \\
Nicotine consumption & 9 & 116 & 0.07 \\
NSAID & 4 & 21 & $0.04^{*}$ \\
Corticoids & 3 & 9 & $0.04^{*}$ \\
Leucocytes $>10,000 / \mathrm{mm}{ }^{3}$ & 5 & 43 & $0.02^{*}$ \\
Haemoglobin $<10 \mathrm{~g} / \mathrm{dl}$ & 11 & 57 & $0.01^{*}$ \\
Urea $>40$ mg\% & 7 & 31 & $0.03^{*}$ \\
Total bilirubin $>5$ mg/dl & 6 & 26 & $0.01^{*}$ \\
Glycaemia $>150$ mg\% & 5 & 22 & $0.02^{*}$ \\
Serum albumin $<3 \mathrm{~g} / \mathrm{dl}$ & 5 & 57 & $0.001^{*}$ \\
Neoadjuvant chemotherapy & 2 & 24 & 0.73 \\
Neoadjuvant radiotherapy & 1 & 37 & 0.56 \\
Infusion solutions $>1000$ ml & 11 & 115 & $0.03^{*}$ \\
Length of intervention $>6$ hours & 9 & 79 & $0.04^{*}$ \\
\hline
\end{tabular}

*factors found to be significant

intra-abdominal collections near the anastomosis or generalized peritonitis or intraoperative anastomosis dehiscence.

We considered the following parameters: gender, age, Charlson comorbidity index, aetiology, alcohol or tobacco consumption, chronic administration of non-steroidal antiinflammatory drugs (NSAIDs) or corticosteroids, leukocyte value $\left(>10,000 / \mathrm{mm}^{3}\right)$, haemoglobin $(<10 \mathrm{~g} / \mathrm{dl})$, urea $(>40$ $\mathrm{mg} \%)$, total serum bilirubin $(>5 \mathrm{mg} / \mathrm{dl})$, glucose $(>150$ $\mathrm{mg} \%)$, albumin $(<3 \mathrm{~g} / \mathrm{dl})$ post neoadjuvant therapy status (chemotherapy, radiotherapy), type of suture (mechanical/ manual), the amount of infusion substance (Ringer's solution, glucose) administered intraoperatively $(>1000 \mathrm{~mL})$, and the duration of the surgical intervention (over $6 \mathrm{~h}$ ).

The Charlson comorbidity index was calculated for each patient included in the study according to the method presented by Hall and Ramachandran [4].

Alcohol consumption was defined as more than $50 \mathrm{~g} /$ day used daily. Tobacco consumption was defined as more

Table III. Calculating the prognostic score of anastomotic fistulas

\begin{tabular}{l}
\hline Prognostic factor \\
\hline Age $>60$ years \\
Malignant aetiology \\
Charlson score $>5$ \\
NSAID \\
Corticoids \\
Leucocytes $>10,000 / \mathrm{mm}^{3}$ \\
Haemoglobin $<10 \mathrm{~g} / \mathrm{dl}$ \\
Urea $>40 \mathrm{mg} \%$ \\
Total bilirubin $>5 \mathrm{mg} / \mathrm{dl}$ \\
Glycaemia $>150 \mathrm{mg} \%$ \\
Serum albumin $<3 \mathrm{~g} / \mathrm{dl}$ \\
Infusion solutions $>1000 \mathrm{ml}$ \\
Length of intervention $>6$ hours
\end{tabular}

* Each factor is assigned a point, the final score being the sum of these points.
Table II. Frequency of anastomotic fistulas depending on the main operator

\begin{tabular}{lcc}
\hline Main operator & $\begin{array}{c}\text { Number of } \\
\text { interventions }\end{array}$ & $\begin{array}{c}\text { Number of anastomotic } \\
\text { fistulas }\end{array}$ \\
\hline A & 25 & 1 \\
B & 43 & 2 \\
C & 105 & 12 \\
D & 69 & 5 \\
E & 56 & 3 \\
\hline
\end{tabular}

than 10 cigarettes/day, every day, for more than 1 year. Consumption of NSAIDs and corticosteroids was considered present when patients admitted to having used them for a period of at least one month before the time of surgery. The values of the biological constants studied were those recorded on admission.

The data were obtained from the case report forms of the patients and surgery protocols.

Statistical analysis was performed using the R-Commander programme Version 1.9-2. To analyze the relationship between variables we used logistic regression. A p value below 0.05 was considered to be statistically significant.

\section{Results}

Of the 298 patients included in the study, 125 were female and 173 male, with a mean age of 48.5 years (range between 35 and 83 years). Benign aetiology was found in 93 cases and malignant aetiology in 205. From a histopathological point of view, colon adenocarcinomas were predominant. Ileo-colic anastomosis was performed in 61 cases, colo-colic anastomosis in 107 cases, and colo-rectal in the remaining $130.31 .98 \%$ of the patients benefitted from protective ileostomy.

Anastomosis in the patients included in this study was performed using single-layer $(\mathrm{n}=75)$ and double-layer ( $\mathrm{n}$ $=150)$ manual sutures, as well as mechanical sutures $(\mathrm{n}=$ 73).

$68.7 \%$ of the cases included in the study had associated pathology, quantified with the Charlson comorbidity index. Cardiovascular diseases predominated (history of acute myocardial infarction, heart failure, peripheral vascular diseases), encountered alone or in combination with obesity, insulin dependent diabetes, chronic consumption of alcohol and nicotine. We considered that a Charlson score greater than 5 is a potential risk factor for the development of anastomotic complications.

Table I shows the distribution of anastomotic fistulas according to the possible adverse prognostic parameters. There were no statistically significant differences between patients in which anastomosis was performed using mechanical suture and those in which anatomosis was performed using manual suture $(\mathrm{p}=0.91)$. When comparing the groups who benefited from protective ileostomy and those without protective ileostomy, the "p" value obtained was 0.45 , statistically insignificant. The differences between the 2 groups were noticed only in terms of the clini- 
cal manifestations in patients who developed postoperative anastomotic dehiscence and the influence on their general health condition.

In the study conducted, postoperative digestive fistulas had a frequency of $7.71 \%$. Of the 23 cases, 15 were found in males and 8 in females, with a mean age of 63.7 years, while mortality in the group of patients with anastomotic dehiscence amounted to $21.72 \%$.

Other postoperative complications were also recorded, namely: suppuration of the wound $(\mathrm{n}=31)-$ the most frequent, respiratory complications $(n=7)$, urinary complications $(\mathrm{n}=5)$, deep vein thrombosis $(\mathrm{n}=2)$, complications of the stoma $(\mathrm{n}=11)$, septic shock $(\mathrm{n}=8)$.

The average length of hospitalization was 10.1 days for patients who did not develop postoperative anastomotic fistula and 35.7 for those with anastomotic dehiscence.

During the 12 years that were studied, we identified five teams who performed the surgical interventions. As shown in Table II, there are no statistically significant differences regarding fistulas and mortality rate $(\mathrm{p}=0.67)$. The mean duration of surgery was 4.1 hours, with no significant differences among the 5 surgical teams.

The factors that have been found to be statistically significant were included in a prognostic score for quantifying the risk of developing anastomotic dehiscence. Thus, 1 point was assigned to each negative prognostic factor, the final score resulting from their sum (Table III). The values recorded in our study were between 0 and 9 (possible values between $0-13$ ). The correlation coefficient between a high prognostic score and development of anastomotic fistulas was 0.75 . The correlation coefficient between the expected prognostic score and the rest of the complications encountered in patients was 0.26 .

\section{Discussions}

Postoperative digestive fistulas are among the most common complications that can occur in surgical services along with suppuration of the wound. Their bad prognosis of the patient's condition, the difficulties of early diagnosis and the optimal choice of the therapeutic method turn them into an issue of great interest. About a third of the deaths occur due to digestive anastomoses following fistulas [4].

The frequency of postoperative digestive fistulas recorded in our study $(7.71 \%)$ is consistent with that of the studies published in the literature, the percentage variations resulting from lack of a clear definition of this topic. However, a percentage of around $10 \%$ is considered to be acceptable.

Male gender was reported as a risk factor in the development of fistulas, which has not been confirmed by the study conducted in our clinic. However, we could demonstrate that age over 60 years correlated with a bad prognosis, most likely due to the association of significant comorbidities in this category.

The use of the Charlson comorbidity score as a prognostic factor of postoperative digestive fistulae is an innova- tion, as there are no other published studies in this regard. The Charlson comorbidity index predicts the ten-year mortality for a patient who may have a range of comorbid conditions, such as heart disease, AIDS, or cancer (a total of 22 conditions). Each condition is assigned a score of 1 , 2,3 , or 6 , depending on the risk of dying associated with each one. Scores are summed to provide a total score to predict mortality $[6,7]$.

Chronic use of NSAIDs and corticosteroids influences digestive healing by decreasing the inflammatory properties of the body that speed the healing process. Moreover, corticosteroids act by reducing epithelisation and neovascularisation $[2,8]$.

Jaundiced patients have a higher rate of gastrointestinal anastomoses compared with the other groups. Experimental studies in rats have shown that an increase in the serum bilirubin resulted in a reduction of collagen synthesis [2].

Unbalanced diabetes affects digestive anastomosis healing through the hypoxia caused by microangiopathy and macroangiopathy, insulin being also important in the early stages of healing.

The use of protective ileostomy was not found to yield statistically significant benefits. This has proved its superiority for low colorectal anastomosis by decreasing the frequency of fistulas and reducing the number of reinterventions and adverse consequences in the event of such complications $[9,10,11]$. Other methods mentioned in the literature to reduce the incidence of anastomotic dehiscence are the use of biological tissue glue, first mentioned by Collet and Rabaud in 1992 as a method of treatment of digestive fistulas, showing its effectiveness in increasing resistance of digestive sutures [12,13]. In 2005 in the Journal of Surgical Research Yin I reminds of the use of peritoneal graft as a method of treatment of major sero-muscular defects in the small intestine, considering it to be a simple, efficient, feasible and economic method [14,15]. He also showed the effectiveness of omentoplasty in reducing the incidence of digestive fistulas. However, all these methods lack randomized clinical trials $[16,17,18]$.

Reports in the literature with regard to the suture material showed that all types of threads produce an inflammatory response, none of them being superior to the other, and that future studies are required, given that a reduced inflammatory response at the level of the suture could lead to a better healing of the anastomosis $[19,20]$. Other studies compare the suture techniques in terms of eversion vs. inversion of the suture sections, in one layer vs. two layers, interrupted or continuous, mechanical or manual sutures, with variable results $[21,22]$. A higher level of stenosis was demonstrated in the case of suture inversing the margins and in double layer, as well as an increased frequency of fistulas in the case of continuous thread anastomoses $[23,24,25]$. Mechanical suture results in a minimal inflammatory reaction at the level of the sections, with explosion pressure and tensile strength higher than those in the group in which manual sutures were used. Their effect on 
collagen concentration in the anastomosis is not yet known [26].

The inclusion into the study of the data related to the surgical teams removes the possibility that the incidence of such complications could be determined by the surgeon's experience, which, certainly, plays a part, but it does not generate statistically significant differences.

The existence of a risk score to quantify the occurrence of anastomotic fistulas would provide the surgeon the opportunity to determine the best option for treatment of a condition that requires performance of digestive anastomosis, both in terms of the proper choice of the time of surgery and of the therapeutic method. On the other hand, knowing the risk factors may lead to the development of new methods to reduce the incidence of anastomotic complications. The obtained correlation coefficient of 0.75 signifies a very good association between the prognostic score and the occurrence of anastomotic dehiscence, while the value of 0.26 obtained in the case of other complications suggests an acceptable combination.

We can say that the purpose of our study was achieved, this being a first attempt to determine a prognostic score of postoperative fistulas. The particularity of the study resides in the specific analysis of each risk factor and in the inclusion of all parameters that have proven to be involved in the development of fistulas in the calculation of the score.

A drawback of the study is its retrospective nature, a prospective study being necessary in order to establish the real value of this score in clinical practice.

\section{Conclusions}

Age over 60 years, malignant aetiology, associated significant comorbidities and chronic consumption of NSAIDs and corticosteroids increases the risk of developing postoperative digestive fistulas. Among the biochemical parameters influencing intestinal healing are: blood glucose, urea, serum albumin, total bilirubin, leukocytes and haemoglobin. Administration of more than $1000 \mathrm{ml}$ during surgery and duration of the surgical intervention of more than 6 hours is also a factor of poor prognosis. Our study demonstrated that the score resulting from the combination of these factors is a reliable method for predicting digestive fistulas.

\section{References}

1. Zaharie $F$, Mocan $L$ et al. Factorii de risc ai fistulelor anastomotice după chirurgia neoplaziilor colo-rectale. Chirurgia. 2012;107:27-32.

2. Sârbu-Boeți MP. Actualități despre fistulele digestive postoperatorii. Etiopatogenie, diagnostic și tratament. Editura Celsius. București, 2006

3. Calu V, Duțu M, Dumitrescu I, et al. Fistulele gastrice postoperatorii asociate cu sepsis sever. J Rom Anest Terap Int. 2010;17:55-60.
4. Gluszek S, Korczack M, et al. Digestive system fistula - a problem still relevant today. Przeglad Chirurgiczny. 2011;83(1):32-41.

5. Kuman A, et al. Anterior resection: anastomotic leaks and strictures. World J Gastroenterol. 2011;17(11):1475-9.

6. Quan $\mathrm{H}$, Li B, Couris CM, et al. Updating and validating the Charlson comorbidity index and score for risk adjustment in hospital discharge abstracts using data from 6 countries. Am J Epidemiol. 2011;173(6):67682.

7. Tan WP, Talbott VA, Leong QQ, Isenberg GA, Goldstein SD, et al. American Society of Anesthesiologists class and Charlson's comorbidity index as predictors of postoperative colorectal anastomotic leak: a singleinstitution experience. J Surg Res. 2013;184(1):115-9.

8. Selvasekar CR, Cima RR, Larson DW, et al. Effect of infliximab on shortterm complications in patients undergoing operation for chronic ulcerative colitis. J Am Coll Surg. 2007;204(5):956-62.

9. Bugiantella W, Rondelli F, Mariani L, et al. Traditional lateral ileostomy versus percutaneous ileostomy by exclusion probe for the protection of extraperitoneal colo-rectal anastomosis: The ALPPI (Anastomotic Leak Prevention by Probe lleostomy) trial. A randomized controlled trial. Eur $\mathrm{J}$ Surg Oncol. 2014 Jan 24. doi: 10.1016/j.ejso.2013.12.025

10. Monzón-Abad A, Gracia-Roche C, Martínez-Germán A, BarrancoDomínguez I, Sánchez-Fuentes N. A preliminary study of transcaecal ileostomy as an alternative to defunctioning ostomies. Colorectal Dis. 2014;16(2):130-3.

11. Vallribera Valls F, Villanueva Figueredo B, Jiménez Gómez LM, et al. lleostomy closure in a colorectal surgery unit. Comparative analysis of different techniques. Cir Esp. 2014 Jan 9. doi: 10.1016/j. ciresp.2013.04.021

12. Victorzon M, Victorzon S, Peromaa-Haavisto P. Fibrin glue and stents in the treatment of gastrojejunal leaks after laparoscopic gastric bypass: a case series and review of the literature. Obes Surg. 2013 Oct;23(10):1692-7.

13. Yin WY. Free peritoneal graft for repair of severe seromuscular defect of bowel: from experiment to clinical practice. J Surg Res. 2005;125(1):3-8.

14. Chang D, Zhang $Y$, et al. Prevention of anastomotic leakage after low anterior resection in rectal cancers. Hepatogastroenterology. 2010;57(99100):477-81.

15. Manusch $F$, Koch $A$, et al. Value of a protective stoma in low anterior resections for rectal cancer. Dis Colon Rectum. 2002;45(9):1164-71.

16. Husu N, Michalski CW. Systematic review and meta-analysis of the role of defunctioning stoma in low rectal cancer surgery. Ann Surg. 2008;248(1):52-60.

17. Slieker JC, Daams F, Mulder IM, Jeekel J, Lange JF. Systematic review of the technique of colorectal anastomosis. JAMA Surg. 2013;148(2):190-201.

18. Lee WL, Epstein KL, Sherlock CE, Mueller PO, Eggleston RB. In vitro comparison of a single-layer (continuous Lembert) versus two-layer (simple continuous/Cushing) hand-sewn end-to-end jejunoileal anastomosis in normal equine small intestine. Vet Surg. 2012;41(5):589-93.

19. Chen C. The art of bowel anastomosis. Scand J Surg. 2012;101(4):23840.

20. Aslan A, Temiza M, et al. Effect of mesalamine on healing in experimental colonic anastomosis: a randomised experimental study. Int J Surg. 2008;6(1):40-4.

21. Higa OH, Parra ER, Ab'Saber AM, et al. Protective effects of ascorbic acid pretreatment in a rat model of intestinal ischemia-reperfusion injury: a histomorphometric study. Clinics. 2007;62(3):315-20.

22. Karatepe O, Kurtulus I, et al. Adrenomedulline improves ischemic left colonic anastomotic healing in an experimental rodent model. Clinics. 2011;66(10):1805-10.

23. Ho YH, Ashour MA. Techniques for colorectal anastomosis. World J Gastroenterol, 2010;16(13):1610-21.

24. Schietroma M, Carlei F. Colorectal infraperitoneal anastomosis: the effects of perioperative supplemental oxygen administration on the anastomotic dehiscence. J Gastrointestinal Surg. 2012;16:427-34.

25. Adas G, Arikan S, et al. Mesenchimal stem cells improve the healing of ischemic colonic anastomoses (experimental study). Langenbecks Arch Surg, 2011;396:115-26.

26. Pascual I, Fernandez de Miguel G, et al. Biosutures improve healing on experimental weak colonic anastomoses. Int $J$ Colorectal Dis. 2010;25:1447-51. 